\title{
Swift swimming reef fish as hosts of small juvenile sharksuckers
}

Received: 21 March 2010/ Accepted: 17 June 2010/Published online: 15 July 2010

(C) Springer-Verlag 2010
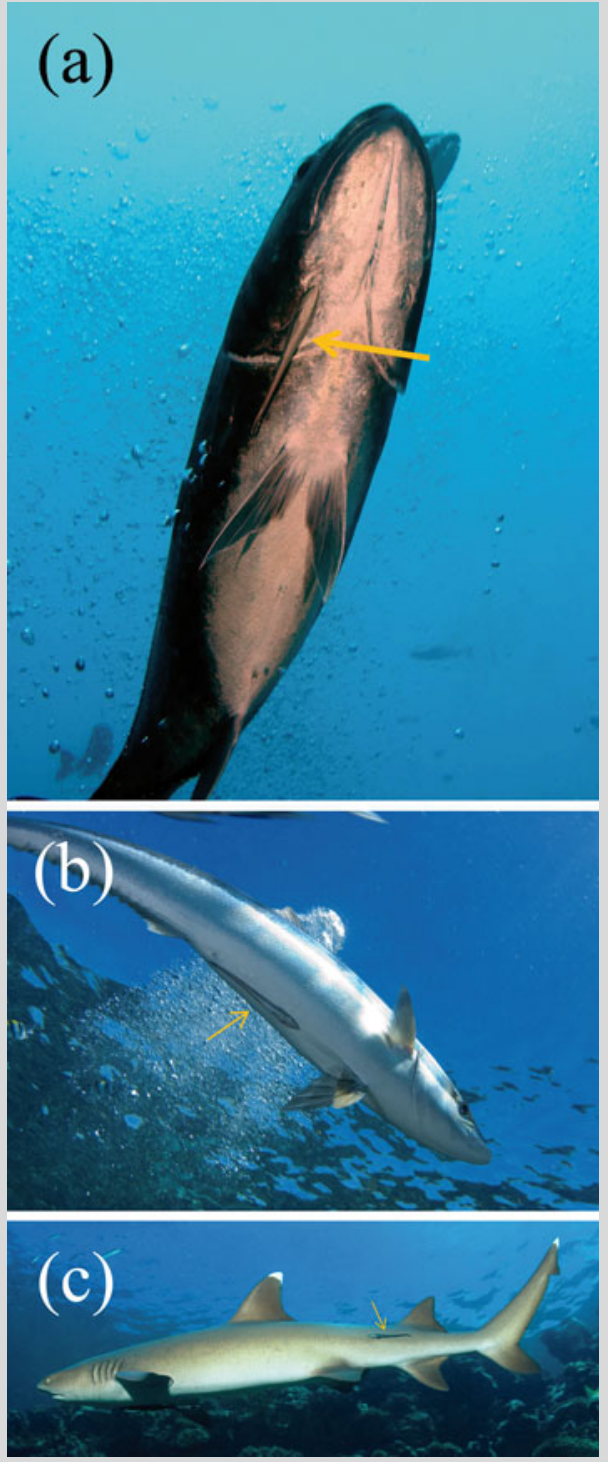

Fig. 1 Juvenile Echeneis naucrates $(<10 \mathrm{~cm})$ attached to Caranx ignobilis (a), Elagatis bipinnulata (b), and Triaenodon obesus (c). Sharksucker size was visually estimated against the host size
Sharksuckers, Echeneis naucrates, attach to a wide variety of hosts (O'Toole 2002; Brunnschweiler and Sazima 2008). Small juvenile sharksuckers are thought to first attach to relatively small and sluggish hosts before they switch to larger, faster moving fish. Here, we report that also larger and fast swimming reef fish such as jacks and sharks serve as hosts for small juvenile E. naucrates, as recorded in the Shark Reef Marine Reserve, Fiji (Brunnschweiler and Earle 2006). Between January and March 2008 and in February 2009 and 2010, small $(<10 \mathrm{~cm})$ sharksuckers were observed attached to the carangids Caranx ignobilis (Fig. 1a) and Elagatis bipinnulata (Fig. 1b) on 77 and $19.7 \%$ of sampling days $(n=61)$, respectively (video footage of sharksuckers attached to a variety of host species including $C$. ignobilis and E. bipinnulata can be found at http://www.explorers-log.com/ by typing in E. naucrates in the search box). Small E. naucrates were also recorded on the snapper Lutjanus bohar (26.2\%) and occasionally on the shark Triaenodon obesus (Fig. 1c). These records indicate that small juvenile sharksuckers use a much broader variety of reef associated fish as hosts, including the swift swimming carangids. We attribute the fact that so far juvenile individuals have only been recorded on relatively sluggish fish species-which have been termed 'trial vehicles' (Strasburg 1964; Brunnschweiler and Sazima 2008)- to the general problem of detecting small remoras, in particular attached on fast-moving hosts. To our knowledge, no definition or concept of trial vehicle for remoras exists and we thus suggest avoiding this term in the future.

Acknowledgments We are grateful to L. Haugen and K. Jost for providing the photographs. We thank the Save Our Seas Foundation, the Shark Foundation Switzerland and Beqa Adventure Divers for financial and logistical support. Three anonymous referees helped to improve the text.

\section{References}

Brunnschweiler JM, Earle JL (2006) A contribution to marine life conservation efforts in the South Pacific: The Shark Reef Marine Reserve, Fiji. Cybium 30(suppl.):133-139

Brunnschweiler JM, Sazima I (2008) A new and unexpected host for the sharksucker (Echeneis naucrates) with a brief review of the echeneidhost interactions. Marine Biodiversity Records 1:e41

O'Toole B (2002) Phylogeny of the species of the superfamily Echeneoidea (Perciformes: Carangoidei: Echeneidae, Rachycentridae, and Coryphaenidae), with an interpretation of echeneid hitchhiking behaviour. Can J Zool 80:596-623

Strasburg DW (1964) Further notes on the identification and biology of echeneid fishes. Pac Sci 18:51-57

J. M. Brunnschweiler ( $\bowtie)$

ETH Zurich, Raemistrasse 101, 8092 Zurich, Switzerland

e-mail: juerg@gluecklich.net

\section{Sazima}

Museu de Zoologia, Universidade Estadual de Campinas, Caixa Postal 6109, Campinas, São Paulo 13083-970, Brazil

Coral Reefs (2010) 29:843 